\title{
Olecranon Bursitis Causing Reflex Sympathetic Dystrophy Syndrome in a Hemiplegic Extremity
}

\author{
Te-Jung Liu ${ }^{1}$, Shao-Chi Lu ${ }^{2}$, Shin-Tsu Chang ${ }^{3 *}$ \\ ${ }^{1}$ Department of Physical Medicine and Rehabilitation, Taoyuan Armed Force General Hospital, Taoyuan, Taiwan (China); ${ }^{2}$ Department \\ of Physical Medicine and Rehabilitation, Hualien Armed Force General Hospital, Hualien, Taiwan (China); ${ }^{3}$ Department of Physical \\ Medicine and Rehabilitation, Tri-Service General Hospital, School of Medicine, National Defense Medical Center, Taipei, Taiwan \\ (China). \\ Email: *stchang@ms87.url.com.tw
}

Received July $11^{\text {th }}, 2012$; revised August $14^{\text {th }}, 2012$; accepted August $20^{\text {th }}, 2012$

\begin{abstract}
Olecranon bursitis is rarely seen in patients with stroke, but reflex sympathetic dystrophy syndrome is not. Here, we report a stroke case, which represented the clinical symptom of reflex sympathetic dystrophy syndrome after development of olecranon bursitis in few days, and describe his clinical association between both conditions. Sonographicpicture of the right elbow in this case is shown.
\end{abstract}

Keywords: Olecranon Bursitis; Reflex Sympathetic Dystrophy Syndrome; Stroke

\section{Introduction}

Olecranon bursitis $(\mathrm{OB})$ is rarely seen in patients with stroke, but in contrast, reflex sympathetic dystrophy syndrome (RSDS) is not true. RSDS is anactual condition definited by localized or diffused pain [1]. The affirmed etiology between RSDS and stroke, the degree of severity and recover of motor deficit with sensory problem was not clear [2]. Meanwhile, there is no universally accepted tools for measuring outcomes inpatients with RSDS are confirmed [3]. RSDS of the upper extremity after stroke is famous as shoulder-hand syndrome. The incidence of shoulder-hand syndrome is varying by diverse clinical data or report [2]. Combination of both conditions has never been reported in that kind of patients yet. We herein report a case of stroke, whose hemiplegic extremity developed olecranon bursitis and ensuing RSDS in a short time.

\section{Case}

A 53-year-old man was admitted to our hospital on 24 December 2010 for inpatient rehabilitation after transference from another hospital following cerebral infarction in the left hemisphere on 23 October 2010. Three weeks after admission, the patient suffered from painful swelling of the right elbow region, which continued to worsen. The local swelling raised with increased temperature in the lesion side (Figure 1). After confirming the diagnosis of $\mathrm{OB}$ clinically, he underwent aspiration

*Corresponding author. of the swelling bursa undersonoguide on 2 January 2011, where showed moderate effusion (Figure 2). Cultures of the aspirated bursal fluid failed to make diagnosis of bacterial and fungal infections.

Shortly after the procedure, the patient grumbled strong sharp pain accompanied withdiffuse swelling of his right upper limb, particularly in the wrist region. The pain worsened while stretching the elbow and wrist joints, which was particularly obvious at night. The diagnosis of RSDS of the right hemiplegic limb was made by physical examination as well as triple phase bone scan, which was performed on 24 January 2011, and revealed mildly to moderately increased tracer uptake during the arterial and soft-tissue phases, and moderately to markedly at the right wrist and MP joints of the right hand (Figure 3).

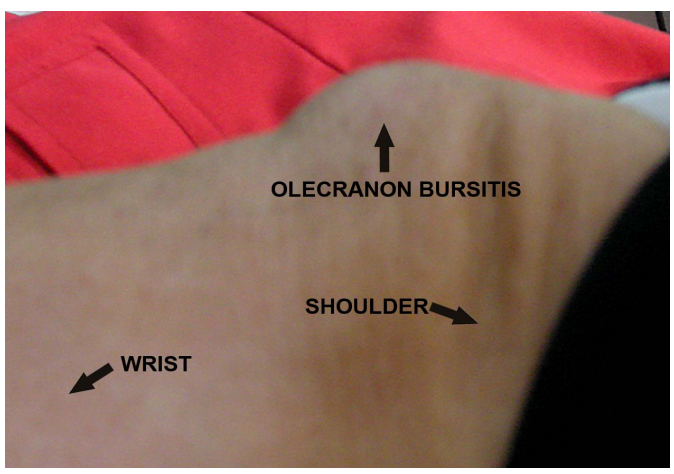

Figure 1. Lateral view of olecranon bursitis of the patient's right elbow in slight flexion. The local swelling of the elbow was shown. 


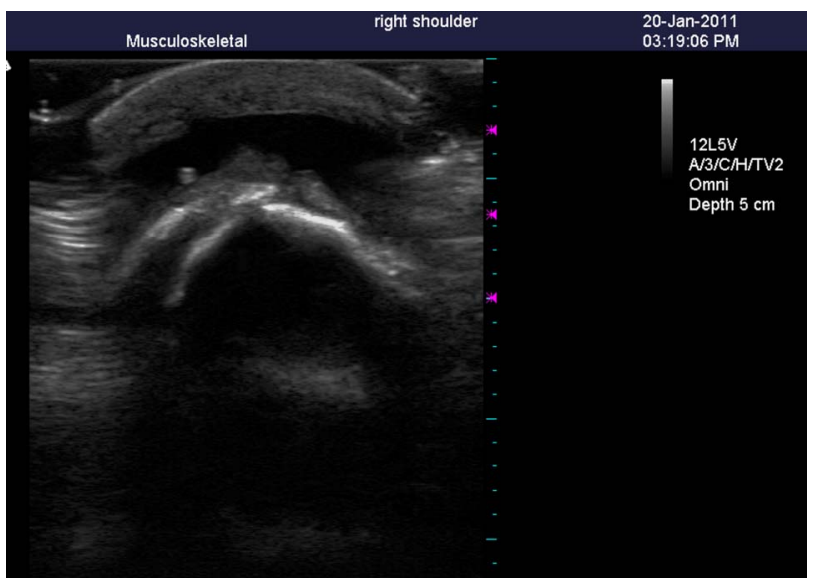

Figure 2. The ultrasonic image of right olecranon bursitisbefore arthocentesis. The bursa was observed to be filled with fluid and was then confirmed laboratorially as aseptic.

Rehabilitation modalitytreated the dystrophic condition with some benefit. Another OB took place in the left elbow one month later, but remitted spontaneously in one week.

\section{Discussion}

This is the first report describing the development of OB and RSDS in the same limb. OB is commonly seen in players in sports, such as wrestling, weight lifting, gymnastics, skateboarding, and rollerblading, particularly when they do not wear elbow protection. OB is also common seen in clinical practice, which etiology can be distinguished as septic and aseptic. There are various kinds of organism resulting in $\mathrm{OB}$, including bacteria, Tubercle Bacillus, fungus, protothecosis etc. The aseptic OB can be related to systemic inflammatory disease, for instance, rheumatoid arthritisor gouty arthritis. According to the literature, the only possible factor contributing to both conditions is infection, but the aspirated bursal fluid failed to confirm the existence of an infection in our patient. The elbow region is the dependent part of the upper limb in supine or seated position, and it may had some degrees of vascular or nerve compromise when it is compressed. We hypothesized that motor deficit might contribute to localized inflammation after repetitive microtrauma, which inflammation result in the subsequent OB.

The peculiar phenomenon of RSDS characterized by autonomic dysfunction and severe pain that may lead to contracture of the affected limbs [4,5]. The incidence for RSDS in the affected paretic upper extremity after stroke varies between $1.5 \%$ and $61 \%$ [6]. Diagnostic criteria include the occurrence of uncommon regional pain and other sensory changes following an injury. RSDS may develop after brain lesions, and also is mostly often commenced by trauma to peripheral nerve or soft tissue [7]. We suggested that an OB lesion would probably

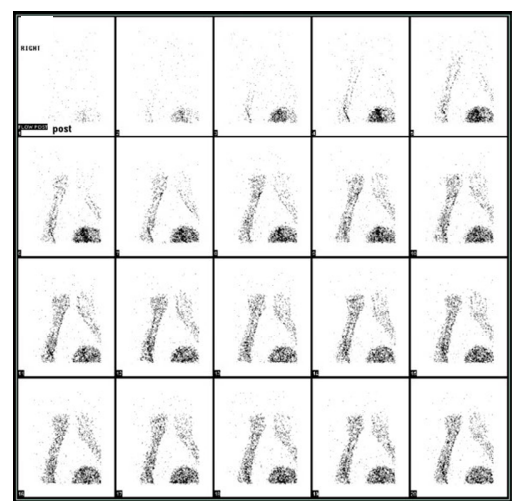

(a)

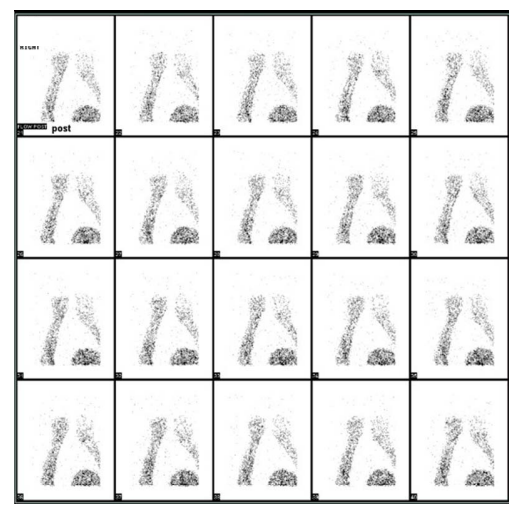

(b)

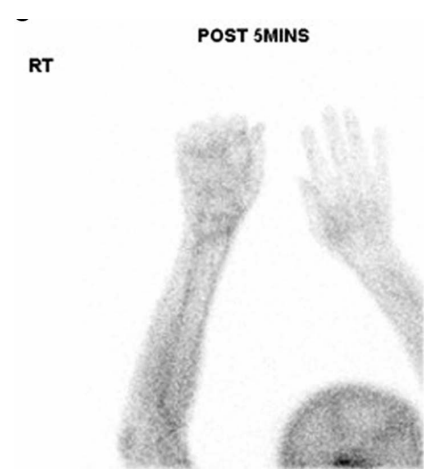

(c)

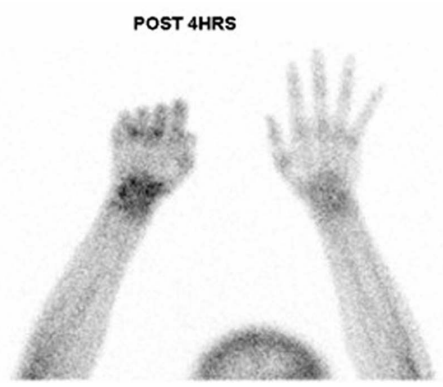

(d)

Figure 3. Images of three-phase bone scan of the right hemiplegic limb. Uptake of Tc-99m was shown increased in bloodpool phase (a), tissue phase (b) and bone phase (c). Stronger isotope uptake can be found in wrist bones and $2 \mathrm{nd}-4$ th metaphalangeal joints in bone phase (d). 
induce RSDS, in some degrees.

The exact cause of RSDS had not been understood well. The development of unilateral sweating abnormalities after stroke led to the hypothesis that autonomic disturbances occurred in stroke and RSDS might share a common central pathophysiology [8]. Important risk factors for development of a RSDS are subluxation, paresis of the shoulder girdle, moderate spasticity and visual deficits for the paretic extremity. Pain is also one of the frequent reasons. A study demonstrated that peripheral traumatic injury of the shoulder joint capsules led to painful extremities after stroke [9]. Some authors claimed that peripheral lesions might initiate a self-perpetuating vicious cycle of pain followed by the full picture of RSDS [10]. We assumed that in stroke patients with extremity $\mathrm{OB}$, like our case, appears to be at a significantly higher risk of developing RSDS. Based on clinical sequence with $\mathrm{OB}$ first and then RSDS in the affected limb, it was difficulty excluding the association between $\mathrm{OB}$ and RSDS and further assumption that RSDS might be induced by $\mathrm{OB}$. We hypothesize that, if $\mathrm{OB}$ develops, the condition should be treated early to prevent the potential development of RSDS.

This report describes the development of RSDS following OB in a hemiplegic limb, and we emphasized that the important role of motor deficit in hemiplegic limb, because it did not happen in the healthy limb even $O B$ occurrence.

\section{REFERENCES}

[1] I. UnlüŞakaci, M. Ersöz, H. Tunç, S. Yilmaz and S. Akkuş, "Simultaneous Complex Regionalpainsyndrome of the Upper and Lower Limb in a Stroke Patient. Case Report," European Journal of Physical and Rehabilitation
Medicine, Vol. 48, No. 1, 2012, pp. 155-161.

[2] S. Pertoldi and P. Di Benedetto, "Shoulder-Hand Syndrome after Stroke. A Complex Regionalpainsyndrome," EuropaMedicophysica, Vol. 41, No. 4, 2005, pp. 283-292.

[3] J. M. Berthelot, "Current Management of Reflex Sympathetic Dystrophy Syndrome (Complexregionalpainsyndrome Type I)," Joint Bone Spine, Vol. 73, No. 5, 2006 , pp. 495-499.

[4] N. K. Gokkaya, M. Aras, E. Yesiltepe and F. Koseoglu, "Reflex Sympathetic Dystrophy in Hemiplegia," International Journal of Rehabilitation Research, Vol. 29, No. 4, 2006, pp. 275-279.

[5] H. Kocabas, F. Levendoglu, O. M. Ozerbil and B. Yuruten, "Complex Regional Pain Syndrome in Stroke Patients," International Journal of Rehabilitation Research, Vol. 30, No. 1, 2007, pp. 33-38.

[6] G. Littlejohn, "Regional Pain Syndrome: Clinical Characteristics, Mechanisms and Management," Nature Clinical Practice, Rheumatology, Vol. 3, No. 9, 2007, pp. 504511.

[7] W. Petchkrua, D. J. Weiss and R. R. Patel, "Reassessment of the Incidence of Complex Regional Pain Syndrome Type 1 Following Stroke," Neurorehabilitation and Neural Repair, Vol. 14, No. 1, 2000, pp. 59-63.

[8] B. Riedl, T. Beckmann, B. Neundörfer, H. O. Handwerker and F. Birklein, "Autonomic Failure after Stroke-Is It Indicative for Pathophysiology of Complex Regional Pain Syndrome?" Acta Neurologica Scandinavica, Vol. 103, No. 1, 2001, pp. 27-34.

[9] D. F. Braus, J. K. Krauss and J. Strobel, "The ShoulderHand Syndrome after Stroke: Aprospective Clinical Trial," Annals of Neurology, Vol. 36, No. 5, 1994, pp. 728-733.

[10] G. Wasner, J. Schattschneider, A. Binder and R. Baron, "Complex Regional Pain Syndrome Diagnostic, Mechanisms, CNS Involvement and Therapy," Spinal Cord, Vol. 41, No. 2, 2003, pp. 61-75. 